This is the peer reviewed version of the following article:

Kassianos, A.P., Coyle, A. and Raats, M.M. (2015) Perceived influences on post-diagnostic dietary change among a group of men with prostate cancer. European Journal of Cancer Care, ISSN (print) 0961-5423, which has been published in final form at http://dx.doi.org/10.1111/ecc.12357. This article may be used for non-commercial purposes in accordance with Wiley Terms and Conditions for Self-Archiving. 


\section{Perceived influences on post-diagnostic dietary change among a group of men with prostate cancer}

Angelos P Kassianos $^{1 *}$, Adrian Coyle ${ }^{2}$, Monique M Raats ${ }^{3}$

${ }^{1}$ Angelos P Kassianos PhD, Research Associate

Primary Care Unit, Department of Public Health and Primary Care, University of Cambridge, UK

${ }^{2}$ Adrian Coyle PhD, Professor of Social Psychology

Department of Psychology, Kingston University, UK

${ }^{3}$ Monique M Raats PhD, Professor and Director of the Food, Consumer Behaviour and Health Research Centre

School of Psychology, University of Surrey, UK

\section{Acknowledgements}

We would like to thank all the men who volunteered their time to participate in this study as well as Professor Margaret Rayman and the Prostate Cancer Charity who helped with recruitment. Also we would like to thank the University of Cambridge, Institute of Public Health's Qualitative Research Forum for their useful comments (especially Katie Mills, Chantal Smeekens and Emily Moran).

\section{Conflict of Interest}

None declared. 
* Corresponding author: Angelos P Kassianos, Worts Causeway, Strangeways Research Laboratory, Cambridge CB1 8RN, UK. Email: ang.kassianos@gmail.com Tel. +44 (0)1223 330323 


\title{
Perceived influences on post-diagnostic dietary change among a group of men with prostate cancer
}

\begin{abstract}
A cancer diagnosis is often associated with loss of agency and control that can adversely affect well-being. Patients may try to regain control through dietary change aimed at preventing progression and/or recurrence. Evidence for the effectiveness of post-diagnostic dietary change in prostate cancer is not conclusive, which can cause uncertainty among patients and health professionals. This qualitative study explored how eight men in the UK, who had been diagnosed with prostate cancer in the previous five years, accounted for any post-diagnostic changes they made to their diet. Data were generated through semi-structured telephone interviews and were subjected to thematic analysis. This yielded two themes concerning the perceived nature and importance of dietary change and the perceived determinants of dietary change. The latter focused on internal dimensions such as agency and external dimensions such as the perceived role of relationships with health professionals, the availability of credible dietary information and family influences. The study points to the importance of the family context in enabling the men to implement dietary change. It is suggested that, even if health professionals can only offer qualified, general advice about diet, this may provide men with a focus for action and a means of regaining control.
\end{abstract}

\section{Keywords}

Agency; dietary change; family; gender; prostate cancer; thematic analysis. 


\section{Perceived influences on post-diagnostic dietary change among a group of men with prostate cancer}

\section{Introduction}

A cancer diagnosis is often associated with a loss of agency, self-efficacy and control which can adversely affect psychological well-being. 'Agency' refers to a person's capacity for making free choices and taking independent action; the related term 'self-efficacy' refers to a person's beliefs about their capacity to take action that influences events in their lives (Bandura, 1982). People who receive a cancer diagnosis and who experience this loss may respond with a desire to exert agency and regain a sense of control through changes in health behaviour at the time of diagnosis and subsequently (Gray et al., 2000; Salminen et al., 2000; Eyre, 2001; Maskarinec et al., 2001). One possible context for health behaviour change is diet.

Prostate cancer largely affects older men, a group that is known frequently to attempt to manage health and illness through food (Pond et al., 2010). The evidence base for the role of diet in prostate cancer prognoses is larger than for many other cancers, although it is difficult to draw confident conclusions (Holick, 2008; Ligibel, 2012). There are indications that dietary modifications after diagnosis can significantly decrease prostate-specific antigen (PSA) levels (the recurrence of the cancer is indicated by high PSA levels) (for example, Dalais et al., 2004); that a diet high in fat is associated with prostate cancer progression and 
mortality (Berkow et al., 2007); and that a diet high in folate (found in some fruits, beans and green vegetables) is associated with lower prostate cancer recurrence (Moreira et al., 2013). Moreover, the consumption of green tea, lycopene (found mainly in cooked tomatoes), omega-3 fatty acids (found mainly in oily fish) and soya has also been linked to reductions in the risk of prostate cancer progression (Davies et al., 2011). However these findings have not been consistently confirmed (Hori et al., 2011).

Due to the inconclusive evidence base, there are no official post-diagnosis dietary guidelines for men with prostate cancer in the UK, although general dietary recommendations are available for people with cancer as a generic group. For example in the UK, Macmillan Cancer Support (2012) advises people with cancer to consume a balanced diet rich in fruit, vegetables and carbohydrates and to reduce meat and dairy consumption and particularly the intake of sugar, salt and foods high in fat. However such guidelines may not be followed. Recent evidence suggests that men diagnosed with prostate cancer may change their diet to facilitate coping (Avery et al., 2014) and prevent recurrence (Satia et al., 2009). Yet, in the USA it has been found that, among groups of men with prostate cancer who were studied, significant proportions did not follow the American Cancer Society’s dietary recommendations about fruit and vegetable intake (Demark-Wahnefried et al., 2000; Blanchard et al., 2004). Despite the lack of official dietary guidelines specifically for men who have had a prostate cancer diagnosis, sources of dietary advice are nonetheless available based on research which has indicated links between diet and prostate cancer prognosis/recurrence. For example, The Prostate Care Cook Book (Rayman et al., 2009) promotes the consumption of cruciferous vegetables, allium vegetables, lycopene, fish, soya, selenium and specific vitamins. In light of these considerations, men diagnosed with prostate 
cancer may face the challenge of making changes to their diet to promote well-being, whether this is based on general healthy eating principles or more specific advice drawn from existing, non-definitive research evidence. In this process, most men will have to deal with health professionals, partners and family members, which may shape their attitudes and emotional responses to their diet and the prospect of dietary change.

Research has pointed to gender-oriented rationales for and patterns of dietary change and management among men with prostate cancer (Mróz et al., 2010, 2011). Generally though, there is relatively little evidence of how men with prostate cancer make sense of changes that they make to their diet post-diagnosis and little is known about the mechanisms of health behaviour change in this specific context (Park et al., 2008). Chapman and Ogden (2009) have suggested that, more generally, people accumulate evidence about diet and health which facilitates their active involvement in diet; the experience of internal and external triggers to action is said to follow from this. The present study responds to the relative scarcity of research literature in this context by qualitatively exploring how a group of men diagnosed with prostate cancer accounted for any changes they made to their diet following diagnosis. More specifically, the aim was to identify factors that they believed were associated with these changes. 


\section{Method}

\section{Participants}

The study was advertised in The Prostate Care Cook Book and in a prostate cancer charity's monthly bulletin following a favourable ethical opinion from the university at which the research was based. Readers who met the eligibility criteria of having received a diagnosis of prostate cancer and being resident in the UK were invited to participate in an online survey and to indicate whether they were interested in being interviewed for this study. All those who expressed an interest were contacted by the first author by to arrange a telephone interview.

Through this process, eight men were recruited and interviewed (see Table 1). Participants were aged between 55 and 76 years, with a mean age of 64.9; all were married or living as married. All had been educated to degree level or above and all were or had been in professional occupations (for example, an architect, a civil engineer, a contracts manager and a social worker). Diagnosis had occurred within the preceding five years. At the time of the study, one participant was in complete remission, two had experienced a recurrence of prostate cancer and the remainder were receiving treatment following their initial diagnosis, with treatment involving surgery or radiotherapy.

[ Insert Table 1 about here ] 


\section{Interview schedule}

The semi-structured interview schedule was based on one used in an earlier study of diet among postmenopausal women who had received treatment for breast cancer (Parry et al., 2009). Participants were asked questions about food choice, perceptions of healthy eating in general and post-diagnosis and whether they had changed their eating habits since they were diagnosed with prostate cancer. An introductory question was used to explore general eating patterns ('Would you describe yesterday as a typical day in terms of what you ate? (If yes) please could you tell me what you ate yesterday, including snacks?'). Any post-diagnostic dietary changes and the rationales for these were explored using a series of questions (for example, 'People decide what to eat in a variety of ways. How do you decide which foods to eat or not to eat?'). Finally, participants were asked about sources of advice about healthy eating in which they had confidence. Interviews were recorded and transcribed.

\section{Analytic procedure}

The interview transcripts were analysed using thematic analysis to generate a set of meaningful patterns or themes associated with the research aims. The analysis followed Braun and Clarke's (2006) six stages of familiarisation with the data, generation of initial codes, searching for themes, reviewing themes (and sub-themes), defining and naming themes and sub-themes, and writing up the analysis. The first author conducted the interviews and analysis initially; the analysis was refined through input from the co-authors. The quality of the analysis was promoted through close alignment with recognised criteria for good qualitative research, such as grounding major interpretations in examples from the data set, conducting credibility checks to avoid idiosyncratic interpretations and optimising coherence across the study (Elliott et al., 1999; Yardley, 2000). 


\section{Results}

Two themes were discerned through the analysis that related to the 'Perceived nature and importance of dietary change' and the 'Perceived determinants of dietary change' (see Table

2). The former theme will be presented briefly to contextualise the latter theme which more explicitly responds to the study's aim of identifying how participants accounted for any postdiagnosis dietary changes. Although the general themes echo the study's aims and, to some extent, the interview topics, the more specific and novel findings occur at the subtheme level.

[ Insert Table 2 about here ]

\section{Perceived nature and importance of dietary change}

Six participants reported having made various changes to their diet after diagnosis,

principally the elimination or reduction of dairy products and red meat, an increased intake of vegetables (especially tomatoes) and the introduction of soya products (soya milk and margarine) into the diet. Some reported an increased intake of fish. The two participants who did not report having made any changes explained this in terms of the perceived healthiness of their diet pre-diagnosis. 
It was evident from participants' accounts that the nature of dietary change centred on changes in participants' relationships with food. Most reported having experienced increased post-diagnosis vigilance in relation to health-enhancing and health-threatening foods and to health and well-being in general. As George said (all participants have been given a pseudonym; ellipsis points indicate where a participant paused in his talk):

It [the diagnosis] made me more aware of certain things I should be definitely be avoiding...reduction in red meat...reduction of dairy products and reduction in sugar.

More specifically the accounts of almost all participants suggested a phase of 'reapproaching' food after diagnosis, which was associated with a re-evaluation of the function, role and meaning of food in their lives. For example, Mark spoke of a shift from regarding and using food as/for consolation before diagnosis to being more aware of its health implications after diagnosis, which he linked to a change in his diet:

My job was particularly stressful so I ate for comfort... Since I was diagnosed I have been more conscious of my weight... and although I am still overweight I am very fit...so I eat very well now...a balanced diet.

To take another example of this sort of shift, Chris talked about his understanding of food having changed from a resource to fulfil physiological requirements pre-diagnosis to a source of justified pleasure now, from unthinking use to mindful, appreciative engagement:

It [diet] is more important. Although I am stricter in my diet, I think actually that I enjoy food more now. It just used to be something you did three times a day - you put the fuel in. Nowadays because I choose and I watch what I eat, I think it is true to say that I enjoy food more now. We can take more care over the preparation of food. I think I appreciate it. I think I took it for granted before and I appreciate it more 
now...We don't really look at the prices any more. We try and buy...you know...the better fruit.

The importance of considering dietary change was evident from participants' reflections. For example, one man spoke of having been prompted by a recurrence of his cancer to consider the importance of diet as an 'obvious' initial context for agentic action:

In a major way but not immediately - only when it came back a year after surgery did I begin to think about what I could do to help myself. And diet was the obvious area to start. (Colin)

\section{Perceived determinants of dietary change}

Participants' perceived determinants of dietary change related to both internal (attitudes) and external (relationships) factors and processes. Four subthemes were identified that suggest what participants believed to be the considerations that may have affected their engagement in dietary change.

The perceived role of relationships with health professionals in influencing dietary action 
All participants indicated that their relationships with health professionals had played a central role in their therapeutic 'journey' after diagnosis and in how they understood their diet in this context. Health professionals' opinions were important to participants post-diagnosis. Mark indicated the importance that he attributed to a health professional's advice as a trigger for health action:

He [the General Practitioner] said, 'It would be helpful if you lost some weight,' so I immediately changed my diet at that point from having being diagnosed to knowing I would have surgery in ten weeks' time.

However it seemed as though participants held high expectations of health professionals' knowledge about diet but at times these expectations were said to have been left unfulfilled. Most participants focused on instances of lack of knowledge on the part of health professionals: they suggested that a lack of information may lead, in one way or another, to individual differences when it comes to changing lifestyle behaviours. One participant believed that health professionals did not consider diet to be an important consideration:

One thing that I would add is that all my contacts with the medical profession, physician or whatever, none of them told me that diet has any relevance at all. (Darren)

However John spoke of having experienced a change in his doctor's outlook on diet in relation to prostate cancer, saying that 'He [the General Practitioner] was a bit sceptical about it [diet] but he has now come around and says, "Well there are some slight indications that it may help."” 
Participants suggested that health professionals' lack of knowledge may lead to patients getting 'the wrong message' about diet and possibly taking inappropriate action. For example, on the basis of his experience, David suggested that a disease prevention and health promotion focus was not sufficiently emphasised:

Well, I went to the doctor and he said, 'Well, ignore it, don't worry and don't bother at all.' And I said to the doctor, 'What about diet?' and he said nothing - 'Diet is useless. It doesn't make a difference. Eat what you like.' And I took the wrong message. So what I thought the doctor said was do what I wanted to do, which was because I felt that this would make me healthier...It [the General Practitioner's reaction] didn't seem to be a healthy living message given and it surprised me that maybe just the attitude of doctors who look for people who are ill and make them better rather than preventative...it didn't seem to be a priority. (David)

\section{Availability of credible dietary information influencing dietary action}

From the participants' perspective, the availability of appropriate and credible information about diet was deemed important in equipping them to make informed changes to their diet. The availability of information per se tends not to be problematic in an age of the internet. For example, Mark said:

Well I have done a lot of research on prostate cancer. After I was diagnosed and before I decided what to eat and to have for my diagnosis, I spent many days on the internet, reading everything I could about all those treatments and what the success ratios were, about impotence and all the other stuff and also a lot of the research on diet. 
Information management seemed to have been a salient concern for participants faced with information that was deemed too general or difficult to evaluate in terms of its evidence base. For example:

Yes, dietary advice is available but not specifically for prostate cancer...There are a lot of different sources aren't there? I mean, there's a lot...published sources are often a lot nowadays. (Darren)

Once you get involved in something you pick up anything you see in the paper or on the television and that is one point I picked up from. (Peter)

In their reflections, some participants spoke of a preference for credible, evidence-based information and used words such as 'facts', 'evidence', 'theories', 'study' and 'researched'. Their talk appeared to be located within a discourse of evidence-based decision-making with a rationalist focus rather than personal experience or appeals to intuition or common sense. For example, David said:

Well what I would feel confident about is whether there would be some evidencebased advice because I think there is probably a lot of...if you look at television and magazines, newspapers, etcetera, there are facts that come and go and I would be more impressed if I could say that there are some researched, evidence-based [facts].

Credible information on diet was considered important for the management of their condition and for reducing the risk of recurrence. Some participants also spoke of seeking accurate information in order to develop explanations for their cancer in terms of past dietary behaviours and thereby render it understandable. For example, Chris said:

If I am to believe what I now believe, that dairy products are a really bad idea as far as breast and prostate cancer goes, that may have been a bad thing because ever since I 
was at the age of 18 I have eaten lots of cheese and drunk lots of milk - a pint of milk a day.

\section{Family influences on dietary action}

In reflecting upon their dietary action post-diagnosis, participants referred to their social roles within their family environments (as partners and parents) as having been influential. Specifically, participants' wives and partners were said to have played an important role in food choice and preparation. There seemed to have been a dependence on partners in relation to dietary change rather than shared decision-making. Some participants attributed this to their partners' specialist knowledge arising from relevant training. For example:

My wife was trained in domestic science and she has, broadly speaking, kept me on a healthy diet with a good variety and plenty of fruit and vegetables. (Darren)

I tend to...my wife tends to take that decision for me [about food]. Well she just says, 'I think we will have some pork chops tonight' and I say, 'Sounds great' and that's the end of it. And I don't know what I will get....It's [His wife's role in determining his diet] probably because my wife is a nurse practitioner. (Mark)

Participants' children were also deemed to have influenced food choice and the control of diet but mostly in the past. Most participants were older men whose children had left home which was said to have facilitated dietary choice post-diagnosis. For example, David said:

Our children grew up and left home and [when they lived at home] they all wanted meat and dairy and all sorts of things which I didn't want to eat... Now when they come and stay with us I don't go and cook the things they want and probably join me 
eating as well. [Family] circumstances made it easier for me to control my diet [postdiagnosis] and so I chose to go a certain way.

\section{Accounts of agency and dietary action}

As can be seen in preceding subthemes and data excerpts, in their accounts of dietary change, most participants described relevant internal psychological processes which can be regarded as forms of agency and self-efficacy. Moving towards the exertion of greater agency in relation to dietary behaviour seemed to have been important for most participants. In relation to his perceived risk of cancer recurrence, David said he felt that 'I should do something about it'; Colin talked of focusing on 'what I could do to help myself'. They expressed a need to feel that what they ate arose from their own decision-making rather than from 'being told to'. The word 'control' appeared in most participants' accounts of what was at stake here and of their rationales for dietary action post-diagnosis. As David put it:

I am quite happy with what I am eating now. It is quite easy for me to control what I eat now so I don't feel pushed to eat something unless I want to.

This type of explanation for action and behaviour was not reported in an unqualified way by all participants, however. For example, Darren said:

You cannot really as a patient...there is nothing you can do about it. You can't control your diet. I felt that I might as well do this and at least I can feel I am doing something towards a cure. Will it be good or not? I don't know... I think it was one and a half or two years [that he maintained his dietary change] but over the past year I have progressively relaxed that [dairy avoidance], returning more or less to a normal 
diet...With certain revelations you are aware of the things she [the health professional] suggested are best avoided.

Here Darren began by connecting his 'patient' status with a strongly-stated position of not having agency about diet, echoing a longstanding and problematized construction of the medical patient as passive (Szasz and Hollender, 1956; Emanuel and Emanuel, 1992). However he went on to describe an intention to change diet as a means of obtaining a sense of some agency in relation to the cancer even though he professes himself uncertain about its effectiveness. This type of qualified, provisional sense of agency is perhaps more typical of people in most cancer trajectories, as is the aspiration for a return to pre-diagnosis normality - seen in Darren's account in his reporting of a progressive return 'more or less to a normal diet'.

Participants did not report comprehensively successful, agentic dietary change, however. Instead many reported having persisted in consuming some foods that were considered unhealthy, although they expressed a wish to adhere more consistently to a healthy diet. The language which some participants used when speaking about this indicated self-recrimination and guilt, with references to 'weakness' and 'frustration'. Others deflected negative selfevaluation by using words such as 'indulgence' and 'naughty', which created a sense of entitlement or forgivable lapses.

Considerations related to the maintenance of dietary change

Although many participants reported having had difficulty in maintaining consistent dietary change, their accounts of change included considerations that could have been positively 
related to change maintenance (or that they explicitly believed had contributed to maintenance). These involved subjective benefits, such as feeling good, and more objective clinical benefits, chiefly low PSA levels. For example, David said:

It seemed to be in the right direction and I know PSA is not very accurate measure but it is something anyway... and in fact the symptoms, which I was having and had, a link with prostate cancer like going to the toilet more often, seemed to disappear when I had a healthier diet.

An ongoing lack of possible recurrence indicators was reported to have been used as an indicator of the value of dietary change and as a motivator for maintaining that change. This occurred even when participants expressed uncertainty about the nature of the relationship between dietary change and non-recurrence. For example, Darren said:

I have no idea whether this dietary discipline is good or not. In fact I am advised that the cancer has probably been cured but it might return so to that extent the treatment and the dietary discipline seem to be effective - but what contribution diet has had, I have no idea. Whether there would have been any difference if I had not taken any notice of my diet, I don't know.

\section{Discussion}

All participants reported either having had a healthy diet pre-diagnosis or having made potentially healthful changes to their diet post-diagnosis in line with research findings on diet and the risk of prostate cancer progression and recurrence. The psychological nature of the 
change centred on participants re-evaluating their relationships with food. Cancer diagnoses often initiate transformations in meaning-making in various life domains. The participant who talked of having re-evaluated food in terms of its intrinsic pleasure typified the sort of renewed mindful awareness that is often reported. However other participants talked of food having been accommodated as a resource within their cancer appraisal and re-evaluated in terms of its health implications. This adds to recent findings that men with prostate cancer change their diets to facilitate coping and for health-enhancing purposes even though they are uncertain of the association between diet and prostate cancer (Avery et al., 2014). The findings also resonated with understandings of health behaviours beyond the prostate cancer context. The perceived determinants of dietary change identified and discussed by participants involved internal and external dimensions, echoing research which has found that healthy individuals experience internal and external triggers to dietary change (Chapman and Ogden, 2009). These dimensions are also evident in theories of health behaviours and responses to 'health threats' (for example, see Leventhal et al., 1998) and in conceptual models that explore the indirect impact of social support on health behaviour change (see Park et al., 2008; Umberson et al., 2010).

Participants spoke in agentic terms about their dietary changes, even if that was sometimes qualified. An emphasis on agency is not simply an artifact of the study: questions about diet were carefully phrased in an exploratory way to avoid suggesting that post-diagnostic dietary change was normative or expected. In some ways, the emphasis on agency was not unexpected, given that cancer diagnoses have been found to be associated with a sense of loss of control (Gray et al., 2000; Salminen et al., 2000; Eyre, 2001; Maskarinec et al., 2001). Also, an emphasis on agency in dietary change is not surprising among a sample of older, 
highly educated, professional men, even allowing for Patterson et al.'s (2003) finding that older people with cancer are less likely to make dietary changes than younger people. Previous research has indicated a positive relationship between high educational level and healthy dietary change (for example, Kristal et al., 2001) and agency has long been represented as a masculine gender-related trait and/or part of a male gender stereotype (see Spence, 1984). Agency has been examined specifically among men with prostate cancer diagnoses. It was found that when agency was accompanied by the ability to express emotions, it was positively linked to men's level of functioning - but not when agency was accompanied by difficulties in emotional expression (Helgeson and Lepore, 1997). The present study was not designed to test such associations. However, among the men in this study, the exertion of agency in relation to diet appeared to have been mediated by contextual, relational considerations, principally where participants had not historically led decision-making about diet in their household. This role had been performed by their female partners, which is typical for this age cohort. This has been said to place men at risk of feminisation when they assume (some) responsibility for making dietary changes in response to a prostate cancer diagnosis (Mróz et al., 2011).

Although it is possible to imagine agentic, health-oriented action having to be compromised to accommodate the dietary preferences of family members, this did not seem to have been the case for the participants in the present study. Instead partners and family members were said to have facilitated a healthy diet through their own choices or at least not to have undermined the men's diet. Yet the men did tend to focus on the role of their partner in implementing changes to their diet. This suggests that what has been termed a 'dyadic', interactive approach to the regulation of diet may have been operating for these participants 
(Karademas and Giannousi, 2013). This is not unusual for men with cancer generally. Previous studies have also identified partners as playing a significant role in men's coping response to cancer and in facilitating lifestyle changes (Helakorpi et al., 2000; Salminen et al., 2000; Badr et al., 2010; Dagan et al., 2011). More specifically, a qualitative study of men with colorectal cancer and their partners concluded that dietary changes were not dependent on the patient alone (Dowsell et al., 2012). This underlines the importance of ensuring that input about diet and other lifestyle issues is disseminated to key members of men's social systems as well as to the men themselves.

Participants offered both positive and negative reflections on the advice about diet received from health professionals. The varied advice that was reported may at least partly reflect the inconclusive nature of the research picture concerning the role of diet in prostate cancer progression and recurrence. This makes it difficult for health professionals to offer definitive guidance to patients. Participants reported having searched for and evaluated relevant information about diet on the internet. Their educational background may have assisted them in evaluating sources but it may be difficult for non-specialists to conduct properly contextualized evaluations. Informed assistance from health professionals may be useful here, even if it can only produce qualified advice about diet. Such advice may be appreciated as it opens up a route to agentic action for patients which may be psychologically beneficial through the promotion or restoration of a sense of self-efficacy. This can apply even in the face of explicitly expressed uncertainty about the role of dietary change in avoiding cancer progression or recurrence, as is seen in the final quotation from Darren. 
In conclusion, the findings of the study apply first and foremost to the group of men who took part and may be at least partly context-specific. Also, when interpreting the findings, it is necessary to consider that two participants were undergoing treatment at the time of their interview, which may have affected their diet. However, the resonances with other research provide evidence for the study having contributed to the development of a progressively comprehensive research picture of considerations relevant to dietary change among men who have been diagnosed with prostate cancer. This offers an evidence base for interventions around diet aimed at promoting well-being through enhancing self-efficacy and coping, equipping support systems, as well as potentially decreasing the risk of cancer progression and recurrence. 


\section{References}

Avery, K.N.L., Donovan, J.L., Horwood, J., Neal, D.E., Hamdy, F.C., Parker, C., Wade, J. and Lane, A. (2014) The importance of dietary change for men diagnosed with and at risk of prostate cancer: A multi-centre interview study with men, their partners and health professionals. BMC Family Practice, 15, 81. DOI:10.1186/1471-2296-15-81.

Badr, H., Carmack, C.L., Kashy, D.A., Cristofanilli, M. and Revenson, T.A. (2010) Dyadic coping in metastatic breast cancer. Health Psychology, 29(2), 169-180.

Bandura, A. (1982) Self-efficacy mechanism in human agency. American Psychologist, $37(2), 122-147$.

Berkow, S.E., Barnard, N.D., Saxe, G.A. and Ankerberg-Nobis, T. (2007) Diet and survival after prostate cancer diagnosis. Nutrition Reviews, 65(9), 391-403.

Blanchard, C.M., Stein, K.D., Baker, F., Dent, M.F., Denniston, M.M., Courneya, K.S. and Nehl, E. (2004) Association between current lifestyle behaviors and health-related quality of life in breast, colorectal, and prostate cancer survivors. Psychology and Health, 19(1), 1-13.

Braun, V. and Clarke, V. (2006) Using thematic analysis in psychology. Qualitative Research in Psychology, 3(2), 77-101.

Chapman, K. and Ogden, J. (2009) How do people change their diets? An exploration into mechanisms of dietary change. Journal of Health Psychology, 14(8), 1229-1242. 
Dagan, M., Saderman, R., Baas, P.C., van Haastert, M., Schokker, M.C. and Wiggers, T. (2011) Spousal support and changes in distress over time in couples coping with cancer: The role of personal control. Journal of Family Psychology, 25(2), 310-318.

Dalais, F.S., Meliala, A., Wattanapenpaiboon, N., Frydenberg, M., Suter, D.A., Thomson, W.K. and Wahlqvist, M.L. (2004) Effects of a diet rich in phytoestrogens on prostate-specific antigen and sex hormones in men diagnosed with prostate cancer. Urology, 64(3), 510-515.

Davies, N.J., Batehup, L. and Thomas, R. (2011) The role of diet and physical activity in breast, colorectal, and prostate cancer survivorship: A review of the literature. British Journal of Cancer, 105, S52-S73.

Demark-Wahnefried, W., Peterson, B., McBride, C., Lipkus, I. and Clipp, E. (2000) Current health behaviors and readiness to pursue life-style changes among men and women diagnosed with early stage prostate and breast carcinomas. Cancer, 88(3), 674-684.

Dowsell, G., Ryan, A., Taylor, A., Daley, A., Freemantle, N., Brookes, M., Jones, J., Haslop, R., Grimmett, C., Cheng, K.K. and Sue, W. (2012) Designing an intervention to help people with colorectal adenomas reduce their intake of red and processed meat and increase their levels of physical activity: A qualitative study. BMC Cancer, 12, 255. DOI:10.1186/14712407-12-255. 
Elliott, R., Fischer, C.T. and Rennie, D.L. (1999) Evolving guidelines for publication of qualitative research studies in psychology and related fields. British Journal of Clinical Psychology, 38(3), 215-229.

Emanuel, E.J. and Emanuel, L.L. (1992) Four models of the physician-patient relationship. Journal of the American Medical Association, 267, 2221-2226.

Eyre, H.J. (2001) Nutritional advice for cancer survivors. CA: A Cancer Journal for Clinicians, 51, 151-152.

Gray, R.E., Fitch, M., Phillips, C., Labrecque, M. and Fergus, K. (2000) Managing the impact of illness: The experiences of men with prostate cancer and their spouses. Journal of Health Psychology, 5(4), 531-548.

Helakorpi, S., Uutela, A., Prättälä, R. and Puska, P. (2000) Health Behaviour and Health among Finnish Adult Population. Helsinki: Publications of National Public Health Institute B8.

Helgeson, V.S. and Lepore, S.J. (1997) Men's adjustment to prostate cancer: The role of agency and unmitigated agency. Sex Roles, 37(3-4), 251-267.

Holick, M.F. (2008) Prostate cancer survival: Is there a dietary connection? Nutrition Reviews, 66(7), 425-426. 
Hori, S., Butler, E. and McLoughlin, J. (2011). Prostate cancer and diet: Food for thought? BJU International, 107(9), 1348-1359.

Karademas, E.C. and Giannousi, Z. (2013) Representations of control and psychological symptoms in couples dealing with cancer: A dyadic regulation approach. Psychology and Health, 28(1), 67-83.

Kristal, A.R., Hedderson, M.M., Patterson, R.E. and Neuhauser, M.L. (2001) Predictors of self-initiated, healthful dietary change. Journal of the American Dietetic Association, 101(7), 762-766.

Levethal, H., Leventhal, E.A. and Contrada, R.J. (1998) Self-regulation, health, and behavior: A perceptual-cognitive approach. Psychology and Health, 13(4), 713-733.

Ligibel, J. (2012) Lifestyle factors in cancer survivorship. Journal of Clinical Oncology, 30(30), 3697-3704.

Macmillan Cancer Support (2012) A healthy eating guide. Available at: http://www.macmillan.org.uk/Cancerinformation/Livingwithandaftercancer/Eatingwell/Healt hyeatingguide/Healthyeatingguide.aspx (accessed 16 May 2014).

Maskarinec, G., Murphy, S., Shumay, D.M. and Kakai, H. (2001) Dietary changes among cancer survivors. European Journal of Cancer Care, 10(1), 12-20. 
Moreira, D.M., Banez, L.L., Presti Jr, J.C., Aronson, W.J., Terris, M.K., Kane, C.J., Amling, C.L. and Freedland, S.J. (2013) High serum folate is associated with reduced biochemical recurrence after radical prostatectomy: Results from the SEARCH Database. International Brazilian Journal of Urology, 39(3), 312-319.

Mróz, L.W., Chapman, G.E., Oliffe, J.L. and Bottorff, J.L. (2010) Prostate cancer, masculinity and food: Rationales for perceived diet change. Appetite, 55(3), 398-406.

Mróz, L.W., Chapman, G.E., Oliffe, J.L. and Bottorff, J.L. (2011) Gender relations, prostate cancer and diet: Re-inscribing hetero-normative food practices. Social Science and Medicine, 72(9), 1499-1506.

Park, C.L., Edmondson, D., Fenster, J.R. and Blank, T.O. (2008) Positive and negative health behavior changes in cancer survivors: A stress and coping perspective. Journal of Health Psychology, 13(8), 1198-1206.

Parry, B., Lawrence, J., Storey, L., Brown, J., Raats, M., Clarke, D., Horton, S., Stilwell, J. and Rainsbury, R. (2009) Investigation of the phytoestrogen intake of a group of postmenopausal women previously treated for breast cancer. Report for the Food Standards Agency. Project Code: T05030. Available at http://www.foodbase.org.uk/results.php?f_category_id=\&f_report_id=359

Patterson, R.E., Neuhouser, M.L., Hedderson, M.M., Schwartz, S.M., Standish, L.J. and Bowen, D.J. (2003) Changes in diet, physical activity, and supplement use among adults diagnosed with cancer. Journal of the American Dietetic Association, 103(3), 323-328. 
Pond, R., Stephens, C. and Alpass, F. (2010) Virtuously watching one's health: Older adults' regulation of self in the pursuit of health. Journal of Health Psychology, 15(5), 734-743.

Rayman, M., Dilley, K. and Gibbons, K. (2009) Healthy Eating: The Prostate Care Cookbook. London: Kyle Cathie Ltd.

Salminen, E.K., Lagstrom, H.K., Heikkila, S. and Salminen, S. (2000) Does breast cancer change patients' dietary habits? European Journal of Clinical Nutrition, 54(11), 844-848.

Satia, J.A., Walsh, J.F. and Pruthi, R.S. (2009) Health behavior changes in white and African American prostate cancer survivors. Clinical Oncology, 32(2), 107-117.

Spence, J.T. (1984) Masculinity, femininity, and gender-related traits: A conceptual analysis and critique of current research. In: Maher BA and Maher WB (eds) Progress in Experimental Personality Research (Vol. 13). San Diego, CA: Academic Press, pp.1-97.

Szasz, T.S. and Hollender, M.H. (1956) A contribution to the philosophy of medicine: The basic models of the doctor-patient relationship. Archives of Internal Medicine, 97, 585-592.

Umberson, D., Crosnoe, R. and Reczek, C. (2010) Social relationships and health behavior across life course. Annual Review of Sociology, 36, 139-157.

Yardley, L. (2000) Dilemmas in qualitative health research. Psychology and Health, 15(2), 215-228. 
Table 1. Participant demographic and medical information

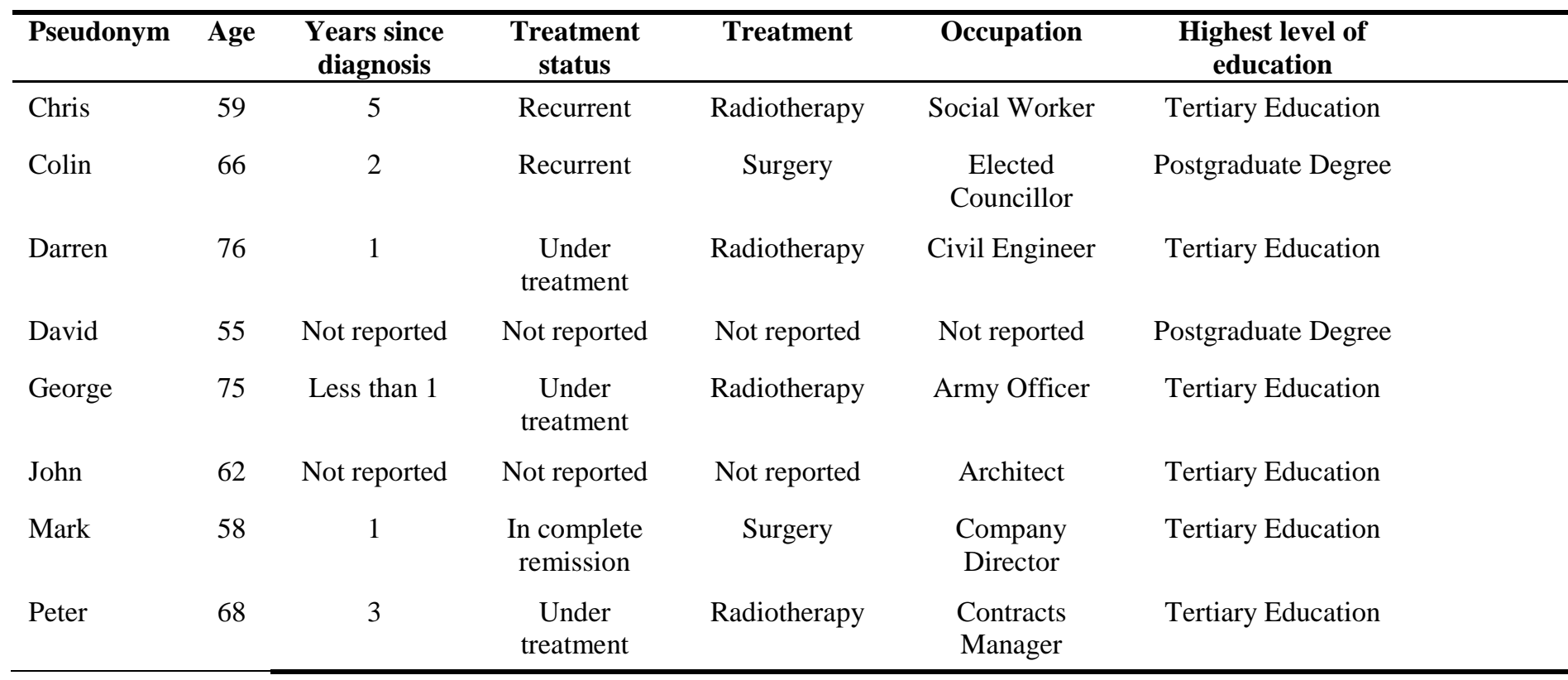


Table 2. Themes and subthemes generated through thematic analysis

\begin{tabular}{|l|l|}
\hline Themes & Subthemes \\
\hline $\begin{array}{l}\text { Perceived nature and importance of } \\
\text { dietary change }\end{array}$ & $\begin{array}{l}\text { The perceived role of relationships with } \\
\text { action professionals in influencing dietary }\end{array}$ \\
\hline Perceived determinants of dietary change & $\begin{array}{l}\text { Availability of credible dietary } \\
\text { information influencing dietary action }\end{array}$ \\
\hline & Family influences on dietary action \\
\hline & $\begin{array}{l}\text { Accounts of agency and dietary action } \\
\text { of dietary change }\end{array}$ \\
\hline
\end{tabular}

